\title{
REANIMATION
}

\section{Besser ohne Mund-zu-Mund-Beatmung?}

Bei der Reanimation durch Laien scheint die zusätzliche Atemspende die Prognose von Patienten mit Herzstillstand aus kardialer Ursache im Vergleich zur alleinigen Herzmassage nicht zu verbessern. Dies bestätigen zwei randomisierte klinische Studien aus Stockholm bzw. aus Seattle und London. In der ersten Studie waren $8,7 \%$ der Patienten nach 30 Tagen noch am Leben, wenn die Reanimation nur durch Brustkompression erfolgte, im Vergleich zu 7\% mit zusätzlicher Atemspen- de. In der zweiten Studie überlebten 12,5\% nach alleiniger Kompressionstherapie und nur $11 \%$ nach Standardreanimation - ein tendenzieller Vorteil. Die inzwischen auch von der American Heart Association ausgegebene Empfehlung „Hands only" für Laienhelfer gilt allerdings nicht für Herzstillstände nach Traumen, infolge von Atemwegsobstruktion oder Asphyxie sowie bei kleinen Kindern. Hier kann die Atemspende Leben retten.

NEJM 2010: 363; 423-33, 434-42, 481

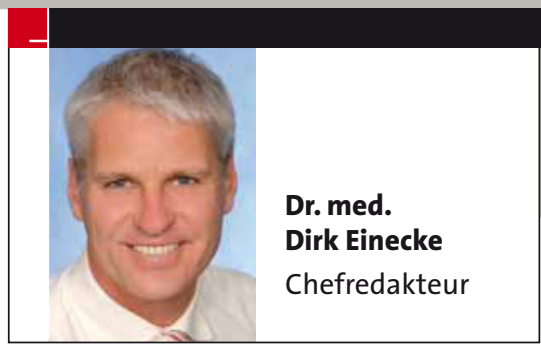

\section{RISKANTE HIGH HEELS}

\section{In die Arthrose gestöckelt?}

Wenn Frauen häufig hochhackige Schuhe tragen, laufen sie Gefahr, ihre Kniegelenke zu ruinieren. Je höher der Absatz, desto größer ist das Risiko für eine Gonarthrose. Darauf weist eine Studie hin, in der bei 15 Frauen untersucht wurde, welche Kräfte beim Laufen auf das Knie einwirken. In High Heels wurde eine verstärkte Kompression des Innenmeniskus festgestellt. Die Belastung stieg mit der Absatzhöhe. Außerdem wurde die Position zahlreicher Ge-

Sprunggelenk modisch verformt. lenke verändert, besonders drastisch war dieser Effekt an den Sprunggelenken. Pressemitteilung lowa State University, August 2010

fisch Kalkablagerungen in den Koronararterien nachweisen. Be einem Ausgangs-LDL < $70 \mathrm{mg} / \mathrm{dl}$ betraf dies nur $8 \%$ der Teilnehmer, bei einem Wert $>160 \mathrm{mg} / \mathrm{dl}$ waren es $44 \%$. Das Risiko für Koronarkalk stieg auch mit sinkendem HDL-Cholesterin. Ein Zusammenhang mit den Triglyzeridspiegeln wurde nicht beobachtet.

Ann Intern Med 2010;153:137-46

\section{Breitere Indikation für MMR-Vakzine}

\section{Neue Impfempfehlungen der STIKO}

Masern: Künftig wird die Masernimpfung (MMR-Impfung) allen nach 1970 Geborenen empfohlen, die nicht oder nur einmal geimpft wurden oder deren Impfstatus unklar ist. Gründe für die erweiterte Empfehlung sind die immer wieder auftretenden Masernausbrüche und die Impflücken bei jungen Erwachsenen.

Influenza: Ab der kommenden Grippesaison wird auch für alle Schwan- geren eine Grippeimpfung empfohlen, da in dieser Patientengruppe häufiger schwere Krankheitsverläufe beobachtet wurden. Die spezielle Impfempfehlung zur pandemischen Influenza hat die STIKO bis auf Weiteres zurückgezogen.

Weitere Infos und einen aktualisierten Impfkalender finden Sie im Epidemiologischen Bulletin 30/2010 unter www.rki.de/impfen.
FRÜHE PRÄGUNG

\section{Mutterliebe schützt vor Stress}

Wer als Kind reichlich Mutterliebe genossen hat, kann als Erwachsener besser mit Stresssituationen umgehen. Das belegt eine prospektive Studie an 482 Personen und ihren Müttern. Die meisten Teilnehmer (85\%) hatten als Kleinkinder Aufmerksamkeit und Zuneigung in normalem Maß erfahren. Nur bei einem von zehn hatte es an Mutterliebe gemangelt. Die übrigen $6 \%$ waren in den Genuss überschwänglicher mütterlicher Zuneigung gekommen. Letztere waren im Erwachsenenalter am wenigsten anfällig für Stress.

Maselko J et al. J. Epidemiol Community Health, doi:10.1136/jech.2009.097873 